\title{
Plastic deformation modes of gallium arsenide in nanoindentation and nanoscratching
}

\author{
K. Wasmer, ${ }^{\text {a) }}$ M. Parlinska-Wojtan, R. Gassilloud, C. Pouvreau, J. Tharian, and J. Micher \\ Empa, Materials Science and Technology, Feuerwerkerstrasse 39, CH-3602 Thun, Switzerland
}

(Received 26 September 2006; accepted 11 December 2006; published online 16 January 2007)

\begin{abstract}
The mechanical deformation by nanoindentation and scratching of gallium arsenide has been investigated using cross-sectional transmission electron microscopy. Twinning was found to be the main deformation process occurring during indentation while only slip bands and perfect dislocations are observed during scratching. This behavior is explained, in the authors' experiments, with the strain rate in scratching being hundred times greater than in indentation. Hence, the low indentation velocity allows twins to be nucleated and propagated from surface inhomogeneities, whereas in scratching, the deformation occurs first in front of the indenter and the scratching speed allows only perfect $\alpha$ dislocation to propagate. (C) 2007 American Institute of Physics.
\end{abstract}

[DOI: $10.1063 / 1.2431763$ ]

Over the last two decades, the mechanical deformation responses of gallium arsenide (GaAs) to contact-induced damage have attracted considerable attention. ${ }^{1}$ Nanoindentation has become the most widespread method to probe via nanocontact testing the mechanical material properties, ${ }^{2}$ to characterize phase transformations ${ }^{3}$ or thin film delamination. ${ }^{4}$ On the other hand, nanoscratching is often used to scrutinize tribological contacts ${ }^{5}$ or to create a defect to analyze the subsequent cleavage and fracture of brittle materials. $^{6-9}$ These studies focused mainly on the scratching effects rather than on the physical phenomena, which have been very little investigated. ${ }^{10,11}$ The main objective of this letter is to compare the deformation mechanisms during nanoindentation and nanoscratching by transmission electron microscopy (TEM).

Most scientists agree that the nucleation of dislocations, also called the elastic-plastic transition, is associated with the pop-in in the load-displacement curve in nanoindentation. ${ }^{12-19}$ Similarly, it is well recognized that the glide planes are $\{111\}$ and the glide directions are $\langle 110\rangle$ leading to the so-called rosette arm patterns. Perpendicular rosette arms exhibit very different shapes and microstructures depending on the material and test conditions. Choi et al. ${ }^{20}$ showed that $\alpha$ dislocations are along the [110] direction with type III (As) atoms in their glide set configuration, whereas $\beta$ dislocations are along [ 110$]$ with type $\mathrm{V}(\mathrm{Ga})$ atoms in their glide set configuration. Each dislocation type has different velocities and mobilities ${ }^{20-24}$ which results in asymmetric rosette patterns. ${ }^{15,21,25}$ However, several authors ${ }^{16,22,26,27}$ raised questions about the $\alpha / \beta$ asymmetry since they did not observed this phenomena. Ambiguous results exist in the literature for the rosette microstructure under indentations on (001) GaAs wafer. Most authors classified their observations in respect to the applied load. This makes any direct comparison between the existing results difficult since the tip geometry and tip radius are different in each experiment. At indentation loads less than $0.55 \mathrm{mN}$, only perfect dislocations are observed. ${ }^{16}$ At indentation loads between 10 and $100 \mathrm{mN}$, perfect dislocations were observed only along
[110], whereas partial dislocations associated with stacking fault and/or twins were only visible in the [110] (Ref. 25-27) direction. On the contrary, for indentations at $50 \mathrm{mN}$, Lefebvre et l. $^{22}$ and Lefevre and Vanderschaeve ${ }^{23}$ saw only perfect dislocation in the [110] direction while twins appeared only in the $[\overline{1} 10]$ direction. Furthermore, microtwins were found to be the dominant deformation mechanisms in indentation at loads ranging from 2 to $100 \mathrm{mN} .^{24,28-30}$ Regarding the origin of the deformation process, there are convincing evidences $^{24,26,27}$ that perfect dislocations are nucleated deeply in the crystal, whereas twins are nucleated on-or very close to- the indented surface due to stress concentrations induced by surface inhomogeneities. Finally, it was shown that microtwinning does not originate from the noncentrosymmetry of the crystal structure but is related to the difference in friction forces acting on the $30^{\circ}(\beta)$ and the $90^{\circ}$ $(\beta)$ partial dislocations. ${ }^{23}$ From this review, it is clear that the deformation and twinning processes of GaAs are still not fully understood. More complete reviews are given in Refs. 1 and 24.

Samples of $5 \times 5 \mathrm{~mm}^{2}$ were cut from a $350 \mu \mathrm{m}$ thick (001)-oriented GaAs wafer with a doping of (1-2) $\times 10^{-18} \mathrm{~cm}^{-3} \mathrm{Si}$ atoms. The nanoindentation and nanoscratching tests were carried out with an MTS Nano-Indenter XP (MTS/Nanoinstruments, Oak Ridge, TN) equipped with a lateral force measurement option. A spherical $(\mathrm{S})$ and a cube corner $(\mathrm{CC})$ tip were employed with tip radii of $\approx 2.7$ and $0.3 \mu \mathrm{m}$, respectively, measured by atomic force microscopy.

The longitudinal TEM lamellae were prepared with a focus ion beam (FIB) FEI Strata DB 235 installation according to Fig. 1 so that a direct comparison between the indentation and scratching deformation process was possible. The microstructure was investigated by TEM. Two microscopes

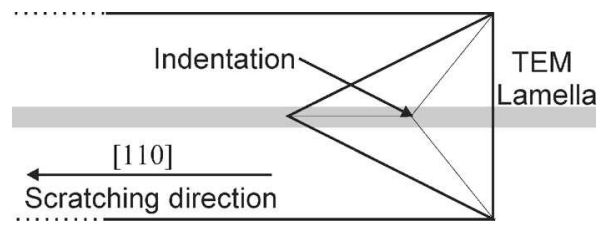

FIG. 1. Top view of the indent and beginning of the scratch.

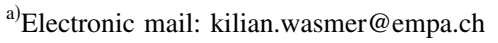




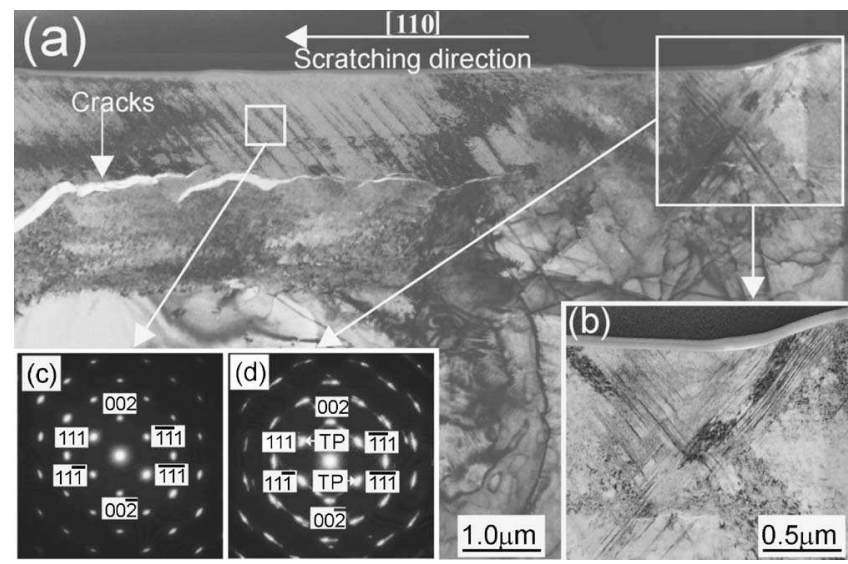

FIG. 2. TEM images of a FIB lamella prepared as a transverse cut through an indent and the beginning of the scratch performed with the spherical indenter: (a) general view (bright field), (b) detailed bright field view of the area under the indent and the beginning of the scratch, and [(c) and (d)] selective area electron diffraction (SAED) patterns taken from the areas under the scratch and the indent, respectively.

were used: a conventional Philips EM430 $\left(\mathrm{LaB}_{6}\right)$ and a Philips CM300 high resolution TEM (equipped with a field emission gun). Their spatial resolutions were 2.5 and $1.8 \AA$, respectively, and the analyses were carried out at an acceleration voltage of $300 \mathrm{keV}$.

Scratches were made along the [110] and [110] directions based on a planar representation given by Sumitomo. ${ }^{31}$ The cube corner tip was oriented with the edge first, as shown in Fig. 1 for the [110] direction. The experiment started by indenting the wafer until the maximum load was reached. Afterward, the tip was moved at constant load making a microscratch. The scratches were $500 \mu \mathrm{m}$ long and performed at a velocity of $50 \mu \mathrm{m} / \mathrm{s}$. To avoid significant damage, the applied loads were 50 and $10 \mathrm{mN}$ for the spherical and cube corner tips, respectively. The corresponding penetration depths during scratching were approximately 0.4 and $1 \mu \mathrm{m}$. The vertical speeds of the indenter during loading, which cannot be controlled but was directly given by the MTS, were $\approx 80$ and $\approx 150 \mathrm{~nm} / \mathrm{s}$.

Figure 2 shows several TEM images for the spherical tip: (a) general view (bright field), (b) zoom of the indent and the beginning of the scratch (bright field), and [(c) and (d)] selective area electron diffraction (SAED) patterns taken under the scratch and the indent, respectively. Figure 3 is similar to Fig. 2 but for the CC tip with an additional SAED pattern (d) at the interface between the residual scratch profile and the bulk. TEM analyses were carried out in [110] and [1110] directions and the deformation microstructure results were identical. Hence, here, only results of scratches in the [110] are presented.

In Figs. 2 and 3, a platinum layer deposited for FIB sample preparation is visible at the top of the surface. The depth of the residual imprint after scratching with the spherical tip is almost constant at $250 \mathrm{~nm}$. In contrast, the deformation caused by the cube corner indenter is much more severe and has a residual depth of approximately $800 \mathrm{~nm}$. Beneath the residual deformation, various microstructural details can be observed ranging from lateral crack formation to dislocations and microtwins. Two main regions can be

distinguished depending on the type of experiment. place. In fact, $\dot{\varepsilon}_{i}$ decreases down to values of 0.22 and
Downloaded 26 Jan 2007 to 129.132.208.23. Redistribution subject to AlP license or copyright, see http://apl.aip.org/apl/copyright.jsp

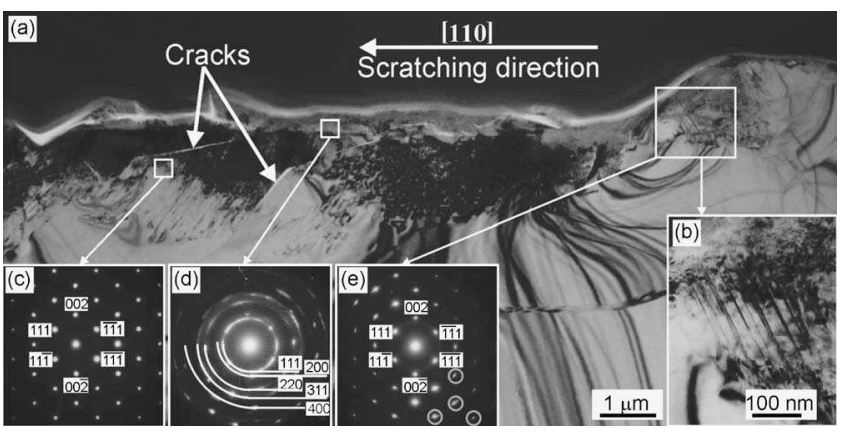

FIG. 3. TEM images of a FIB lamella prepared as a transverse cut through an indent and the beginning of the scratch performed with the cube corner indenter: (a) general view (bright field), (b) detailed bright field view of the area under the indent and the beginning of the scratch and [(c) and (d)] selective area electron diffraction (SAED) patterns taken from the areas under the scratch and the indent, respectively.

First, just under the indent, the mechanism of deformation is a mixture between twinning and dislocation bands. This is clearly demonstrated by the double spots and streaks $^{32}$ visible in the SAED [Figs. 2(d) and 3(e)] taken from the $[\overline{1} 10]$ zone axis. TP in Fig. 2(d) stands for twinning plane. In Fig. 3(e), the double spots are not so obvious, although several of them are visible from the original negative of the SAED, which are encircled for convenience. The small number of double spots observable is certainly due to the small number of twins $(<50)$ shown in Fig. 3(b). These twinning and dislocation bands make an angle of $54.6^{\circ}$ with the surface and so clearly lie in equivalent $\{111\}$ planes.

Secondly, after the scratching operation, it is interesting to notice the presence of lateral cracks as already observed. ${ }^{6,7,17,19}$ Only dislocations and slip bands are observed in this region which is supported by the fact that no double spots are visible on the diffraction pattern but only some streaks ${ }^{32}$ as in Figs. 2(c) and 3(d). In Fig. 2(a), these dislocations and slip bands on the top of the cracks lie, as for twinning, on the (111) plane. The case of the cube corner tip is slightly different since under the scratch an additional pattern is visible and supported by the SAED in Fig. 3(d). Based on this diffraction pattern, there is an extremely high degree of deformation which is due to either a nanocrystalline state, an incoherence in the crystallographic network, or the beginning of an amorphization of the GaAs. ${ }^{33,34}$

From the literature, ${ }^{20-27}$ the occurrence of twinning in indentation is obviously influenced by the tip geometry, the maximum load, and the crystallographic directions. However, the authors believe that the strain rate $\dot{\varepsilon}$, which according to the theory ${ }^{35}$ is related to the macroscopic plastic behavior, plays a vital role in the deformation process. In indentation and scratching, $\dot{\varepsilon}$ is defined, respectively, by

$$
\dot{\varepsilon}_{i}=\frac{1}{h} \frac{d h}{d t} \text { and } \dot{\varepsilon}_{s}=\frac{V_{\text {tip }}}{b},
$$

where $h$ is the depth of the indention, $V_{\text {tip }}$ is the sliding velocity, and $b$ is the groove width. ${ }^{36}$

It is evident from Eq. (1) that, assuming $d h / d t=$ const in an ideal case, $\dot{\varepsilon}$ decreases from an infinite value to the one obtained from the final depth. The infinite value is only theoretical since at the beginning, only elastic deformation takes place. In fact, $\dot{\varepsilon}_{i}$ decreases down to values of 0.22 and 
$0.11 \mathrm{~s}^{-1}$ for spherical and cube corner indenters, respectively. In contrast, $\dot{\varepsilon}_{s}$ is more or less constant during scratching with values of 17.68 and $27.47 \mathrm{~s}^{-1}$ for spherical and cube corner tips, respectively. The strain rate during indentation is, therefore, roughly two orders of magnitude smaller compared to scratching.

Other important parameters to consider are the nucleation sites of perfect dislocations and twins, mentioned earlier, and the dislocation velocities. The maximum velocity of $\beta$ dislocation observed in GaAs is more or less $2.5 \mu \mathrm{m} / \mathrm{s}$, whereas it is hundred times faster for $\alpha$ dislocations. ${ }^{20,24}$ This difference increases with decreasing temperature ${ }^{37}$ Hence, it is believed that twinning arises in indentation for two main reasons. First, the indenter contacts the surface where inhomogeneities exist, leading to stress concentrations. Secondly, the indentation velocity $(<150 \mathrm{~nm} / \mathrm{s})$ is low enough for the nucleation of perfect and partial dislocations which have also time to propagate in packages of several partial dislocations trailing stacking faults which generate twins. However, the tip geometry, the deformation rate, or deformation depth seems to affect the number of twins. It is not clear which of these parameters is responsible since in our experiments, although the cube corner had a load five times less than the spherical tip, its deformation rate, penetration depth, and depth of the residual imprint are larger by a factor between 2 and 3.2. During scratching, the two requirements mentioned above are not fulfilled. In fact, the deformation during scratching occurs first in front of the indenter and it is not the indenter tip which is in contact with the surface but its side. Thus, the effects of surface inhomogeneities become negligible in this process. Furthermore, the scratching velocity $(50 \mu \mathrm{m} / \mathrm{s})$ is much greater than the maximum $\beta$ dislocation $(2.5 \mu \mathrm{m} / \mathrm{s})$ but less than the perfect $\alpha$ dislocations $(250 \mu \mathrm{m} / \mathrm{s})$. This suggests that even if $\beta$ dislocations are nucleated during scratching, they would not be able to move along the different $\{111\}$ planes. In contrast, perfect $\alpha$ dislocations have time not only to nucleate but are fast enough to propagate.

In summary, the deformation processes of GaAs in indentation and scratching have been investigated by TEM. It was found that in all cases, twinning is the main deformation process in indentation, whereas only slip bands and perfect dislocations are seen after scratching. This behavior is explained by the fact that the low indentation velocity allows twins to be generated and propagated from surface inhomogeneities, whereas in scratching the plastic deformation occurs first in front of the indenter and the scratching speed allows only perfect $\alpha$ dislocations to propagate.

The authors are grateful to CIME-EPFL for the using the TEM facility.
${ }^{1}$ E. Le Bourhis and G. Patriarche, Prog. Cryst. Growth Charact. Mater. 47, 1 (2003).

${ }^{2}$ W. C. Oliver and G. M. Pharr, J. Mater. Res. 7, 1564 (1992).

${ }^{3}$ D. Ge, V. Domnich, and Y. Gogotsi, J. Appl. Phys. 93, 2418 (2003).

${ }^{4}$ P. Demarecaux, D. Chicot, and J. Lesage, J. Mater. Sci. Lett. 15, 1377 (1996).

${ }^{5}$ B. Bhushan, Handbook of Micro/Nano Tribology, 2nd ed. (CRC, New York, 1999).

${ }^{6}$ B. Lawn, Fracture of Brittle Solids, 2nd ed. (Cambridge University Press, Cambridge, 1993).

${ }^{7}$ K. Wasmer, C. Ballif, R. Gassilloud, C. Pouvreau, R. Rabe, J. Michler, J.-M. Breguet, J.-M. Solletti, A. Karimi, and D. Schulz, Breast J. 7, 309 (2005).

${ }^{8}$ M. A. Rosentreter, M. Wenderoth, N. H. Theuerkauf, A. J. Heinrich, M. A. Schneider, and R. G. Ulbrich, Europhys. Lett. 38, 675 (1997).

${ }^{9}$ K. Sauthoff, M. Wenderoth, M. A. Rosentreter, K. J. Engel, T. C. G. Reusch, and R. G. Ulbrich, Phys. Rev. B 60, 4789 (1999).

${ }^{10}$ V. Jarder, H. Zahouani, J. L. Loubet, and T. G. Mathia, Wear 218, 8 (1998).

${ }^{11}$ J. L. Bucaille and E. Felder, Philos. Mag. A 82, 2003 (2002).

${ }^{12}$ A. B. Mann and J. B. Pethica, Appl. Phys. Lett. 69, 907 (1996).

${ }^{13}$ A. B. Mann and J. B. Pethica, Philos. Mag. A 79, 577 (1999).

${ }^{14}$ E. Le Bourhis and G. Patriarche, Philos. Mag. Lett. 79, 805 (1999).

${ }^{15}$ E. Le Bourhis and G. Patriarche, Philos. Mag. Lett. 83, 149 (2003).

${ }^{16}$ H. S. Leipner, D. Lorenz, A. Zeckzer, H. Lei, and P. Grau, Physica B 308-310, 446 (2001).

${ }^{17}$ J. E. Bradby, J. S. Williams, J. Wong-Leung, M. V. Swain, and P. Munroe, Appl. Phys. Lett. 78, 3235 (2001).

${ }^{18}$ J. E. Bradby, J. S. Williams, J. Wong-Leung, and S. O. Kucheyev, Philos. Mag. A 82, 1391 (2002).

${ }^{19}$ J. E. Bradby, J. S. Williams, and M. V. Swain, J. Mater. Res. 19, 380 (2004).

${ }^{20}$ S. K. Choi, M. Mihara, and T. Ninomiya, Jpn. J. Appl. Phys. 16, 737 (1977).

${ }^{21}$ P. D. Warren, P. Pirouz, and S. G. Roberts, Philos. Mag. A 50, L23 (1984).

${ }^{22}$ A. Lefebvre, Y. Androussi, and G. Vanderschaeve, Phys. Status Solidi A 99, 405 (1987).

${ }^{23}$ A. Lefebvre and G. Vanderschaeve, Phys. Status Solidi A 107, 647 (1988).

${ }^{24}$ G. Vanderschaeve, Solid State Phenom. 59-60, 145 (1998).

${ }^{25}$ L. Largeau, G. Patriarche, F. Glas, and E. Le Bourhis, J. Appl. Phys. 95, 3984 (2004).

${ }^{26}$ C. Levade and G. Vanderschaeve, Phys. Status Solidi A 171, 83 (1999).

${ }^{27}$ S. Koubaïti, C. Levade, and G. Vanderschaeve, Philos. Mag. A 80, 83 (2000).

${ }^{28}$ S. J. Lloyd, J. M. Molina-Aldareguia, and W. J. Clegg, J. Mater. Res. 16, 3347 (2001).

${ }^{29}$ S. J. Lloyd, A. Castellero, F. Guliani, Y. Long, K. K. McLaughlin, J. M. Molina-Aldareguia, N. A. Stelmashenko, L. J. Vandeperre, and W. J. Clegg, Proc. R. Soc. London, Ser. A 461, 2521 (2005).

${ }^{30}$ F. Giuliani, S. J. LLoyd, L. J. Vandeperre, and W. J. Clegg, in Proceedings of EMAG, Oxford, 2003, edited by S. McVitie and D. McCombe (unpublished), p. 123.

${ }^{31}$ Sumitomo Electric Industries Ltd. Report No. EU-5R, 2005 (unpublished).

${ }^{32}$ J. W. Edington, Practical Electron Microscopy in Materials Science, 2nd ed. (Van Nostrand, Princeton, 1976).

${ }^{33}$ Z. C. Li, L. Liu, X. Wu, L. L. He, and Y. B. Hu, Mater. Sci. Eng., A 337, 21 (2002).

${ }^{34}$ V. A. Pavlov, Phys. Met. Metallogr. 59, 1 (1985).

${ }^{35}$ P. Haasen, Z. Phys. 167, 461 (1962).

${ }^{36}$ B. J. Briscoe and P. S. Thomas, Thin Solid Films 359, 1 (2000).

${ }^{37}$ P. Pirouz, S. Wang, B. Bayu-Aji, M. Zhang, and J.-L. Démenet, Phys. Status Solidi C 2, 1973 (1999). 